\title{
Towards a Stripline Setup to Characterise the Effects of Corrosion and Ageing on the Shielding Effectiveness of EMI Gaskets
}

\author{
D. Pissoort, J. Catrysse,T. Claeys, F. Vanhee, B. \\ Boesman \\ Dept. of Electrical Engineering \\ KU Leuven - Technology Campus Ostend \\ Zeedijk 101, B8400, Ostend, Belgium \\ davy.pissoort@kuleuven.be
}

\author{
C. Brull \\ SEM Schlegel Electronic Materials \\ Slijpesesteenweg 28, \\ B8432 Leffinge, Belgium \\ christian.brull@schlegelemi.com
}

\begin{abstract}
This paper introduces a novel measurement set-up dedicated to the characterization of the evolution of the highfrequency shielding-effectiveness of gaskets due to corrosion and ageing. The measurement set-up is based on the recently introduced stripline set-up which has been validated previously up to $40 \mathrm{GHz}$. Compared to the original stripline set-up, the adapted set-up has a removable "clamping module" which can be easily mounted and removed from the set-up. The clamping module allows to age the gasket inside e.g. a climate chamber while always keeping the gasket under the same compression rate. As the clamping module can be made out of different materials, it allows to study the influence of shieldingeffectiveness of gaskets when applied to different materials. In order to avoid parallel leakage inside the measurement set-up, a high-performant gasket needs to be compressed below the module. Based on full-wave simulations, it is shown that the shielding-effectiveness given by this gasket underneath the clamping module is the main determining factor for the dynamic range of the set-up. In addition, a first set of measurements show the validity of the measurement set-up and approach.
\end{abstract}

Keywords—shielding effectiveness, corrosion, ageing, gaskets, stripline set-up

\section{INTRODUCTION}

Electronic technologies will be key for many upcoming innovations that address major socio/economical challenges. Within the next decade, we will witness, among others, the continuing automation of vehicles (eventually resulting in selfdriving cars), the development of Industry 4.0 which is based on the integration of high-tech electronics in machines, the continuing application of electronics within the medical care, and the rollout of the smart grid. As a direct result, electronic technologies will be used in more sectors and applications where the long-term reliability is a major concern. Moreover, ElectroMagnetic Interference (EMI) will be an important aspect in this long-term reliability because of two reasons. At one hand, the essential building blocks of an electronic system are becoming more vulnerable to EMI because of the lower voltage levels, which are a direct result from miniaturization and the search for energy efficiency. At the other hand, the electromagnetic environment becomes more severe with a larger diversity of EMI covering a very broad frequency spectrum. As an example, the smart grid raises concerns about EMI in the kHz-range, while the application of the anticollision radar in automotive raises concerns about EMI around $80 \mathrm{GHz}$

For the required EMI-hardening, the application of shielding enclosures and gaskets will even become more important than it is today. This applies both to the system level (relatively large enclosures) as to the component level (Board Level Shielding). In combination with the fact that these shielding enclosures and gaskets should maintain their performance over a long lifetime within a possibly harsh environment, this necessitates the development of appropriate test methods that characterize how the shielding performance evolves over time due to e.g. corrosion and ageing.

At this moment, the only suitable method for testing the influence of a hostile environment is included in the SAE ARP 1705 rev. A standard (2006) [1]. SAE ARP 1705 addresses the Transfer Impedance method to provide a figure to characterize the Shielding Effectiveness (SE) of conductive EMI gasket materials in the frequency range up to $1 \mathrm{GHz}$. The transfer impedance method is based on the injection of a current through a sample holder, clamping a gasket. The related voltage drop over the gasket is measured. Although the latest version dates from 2012 (rev. B) and claims an upper frequency of $10 \mathrm{GHz}$, only the previous edition SAE ARP 1705 rev. A allows for the characterisation of the effect on the $\mathrm{SE}$ values due to subjecting a global gasketed joint surface to hostile environments. Consequently, actual environmental testing of the SE of gaskets is restricted to $1 \mathrm{GHz}$.

This paper describes a novel measurement set-up and methodology to characterize the long-term SE of gaskets up to very high frequencies. The method is an adaptation of the stripline method [2]-[5], which has been applied successfully recently to the characterization of the SE of gaskets up to 40GHz. In [6], it was proven that the SE of this stripline method aligns quite well with the SE obtained in a reverberation room. The main adaptation for the 
characterization of the effects of corrosion and ageing comprises a removable "clamping module" which can be easily taken out of the measurement set-up and can be easily mounted in it again. This clamping module allows to age and re-test the gasket while continuously keeping it under the same compression rate. As an additional advantage, the adapted stripline set-up allows to easily study the influence of different contact materials on the gasket performance.

This paper is organized as follows. Section II describes the basics of the stripline method for the characterization of gaskets up to $40 \mathrm{GHz}$ and its definition of the shielding effectiveness. Section III describes in detail the adapted stripline set-up, the mounting of the gasket in the removable clamping module, as well as the mounting of this module in the stripline set-up. Section IV discusses the dynamic range of the adapted set-up based on full-wave simulations. Section $\mathrm{V}$ gives an overview of some preliminary measurement results that prove the validity of the measurement set-up and methodology. Finally, Section V draws concluding remarks.

\section{STRIPLINE METHOD}

\section{A. Measurement Set-Up}

In [7], a method was proposed by Koerber for the characterization of integrated circuits (IC) using a stripline method. This method has been the basis for two IEC standards for characterizing the emission and immunity of ICs [8][9]. The basic principle is shown in Fig. 1. The method is based on putting a 50 Ohm stripline over a PCB board with a full GND layer on top of which the IC under test is mounted.

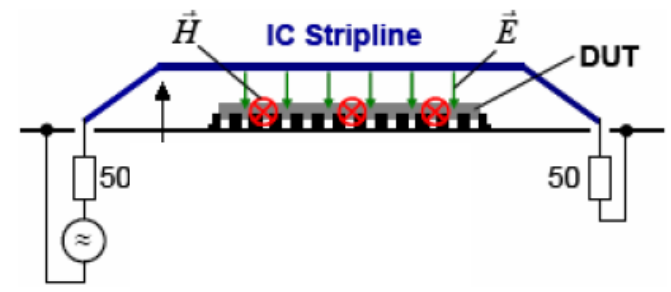

Fig. 1. Set-up for the stripline method for the characterization of ICs

Based on this same approach, a method was proposed in [2][6] to characterize gaskets from $1 \mathrm{GHz}$ up to $40 \mathrm{GHz}$, with special focus on on-board applications. For this frequency range and applications, it is assumed that the dominant coupling path is generated by traces on a PCB and/or the bonding wires of IC packages. In the stripline set-up, this noise source is represented by a $50 \mathrm{Ohm}$ microstrip.

The complete stripline measuring set-up is shown in Fig. 2. The stripline antenna has been designed to have a characteristic impedance of $50 \mathrm{Ohm}$. SMA connectors are mounted through the solid copper ground-plate. The $50 \mathrm{Ohm}$ microstrip representing the on-board noise source is embedded in the ground-plate. Both the embedded microstrip and stripline antenna are terminated in a 50 Ohm resistive load at one end. The other ends are the transmitter and receiver part of the set-up.
Another solid copper plate ('clamping plate') is intended to hold the gasket, and is inserted in the open area of the stripline. In this way, the clamping plate acts as a gasket sample holder, and the gasket can be carefully positioned on the clamping plate (Fig. 3). When placing the clamping plate holder in the stripline set-up, the embedded microstrip is exactly within the inner surface area limited by the gasket.

\section{B. Shielding-Effectiveness}

The SE of the gasket is evaluated by performing two measurements. In the first measurement, the coupling $\left|S_{21, \text { stripline }}^{\text {open }}\right|$ between the microstrip and the stripline antenna is determined for the open structure, i.e. without gasket or clamping plate. In the second measurement, the gasket is mounted onto the clamping plate as shown in Fig. 3. Both are introduced into the stripline set-up. The gasket is compressed up to a predefined level (controlled with some plastic covers of a given and known thickness). Once again the coupling $\left|S_{21 \text {,stripline }}^{\text {gasket }}\right|$ between the microstrip and the stripline antenna is determined. The SE is then defined as the ratio between the two coupling levels:

$$
\mathrm{SE}_{\text {stripline }}=20 \log _{10} \frac{\left|S_{21 \text {,stripline }}^{\text {open }}\right|}{\left|S_{21, \text { stripline }}^{\text {gaske }}\right|} \text {. }
$$

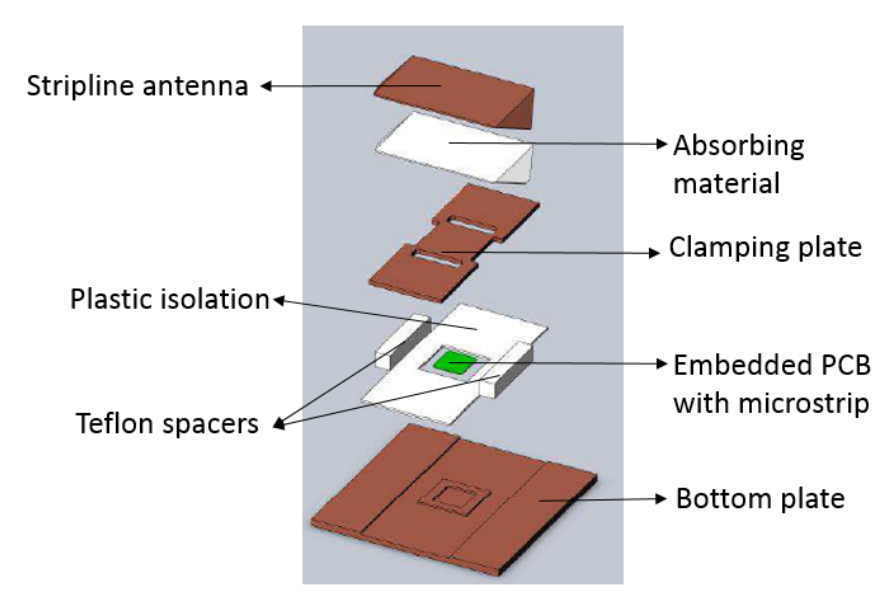

Fig. 2. Overall view of the stripline set-up.

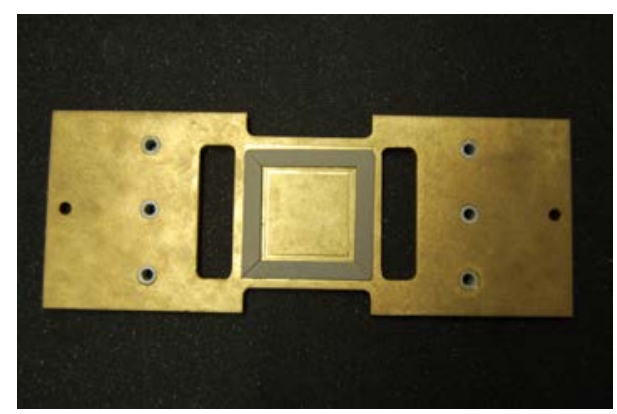

Fig. 3. Mounting of a gasket to the clamping plate. 


\section{ADAPTED TEST FIXTURE}

The stripline set-up described in Section II is not wellsuited for the characterization of the effects that corrosion and ageing have on the gasket's performance. The main disadvantage is that the gasket has to be uncompressed and taken out of the set-up to do the ageing (unless one would put the whole set-up in a climate chamber, which is not practical nor economical). After the ageing, it has to be remounted on the clamped plate and recompressed to the same compression rate. This not only introduces extra uncertainties and possible errors to the measurements of the SE, but also does not allow to study how different contact materials could influence this behavior.

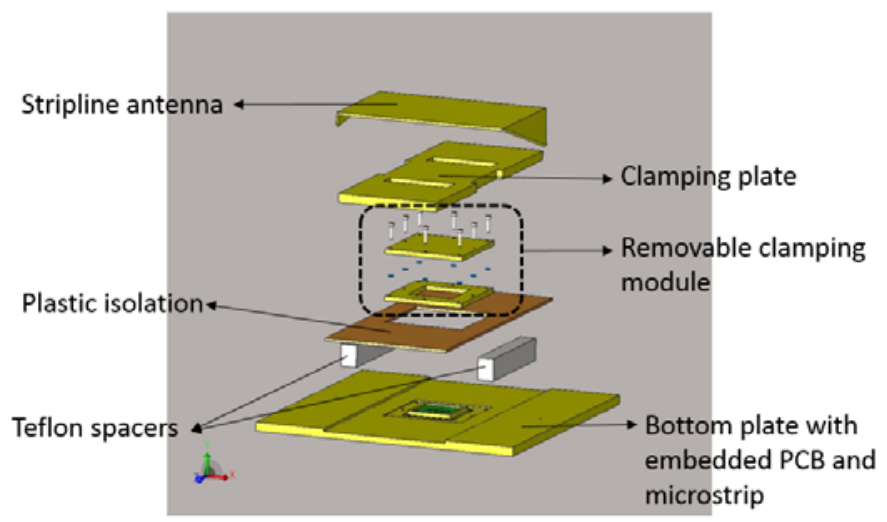

Fig. 4. Overview of the dedicated stripline set-up with removeable module.

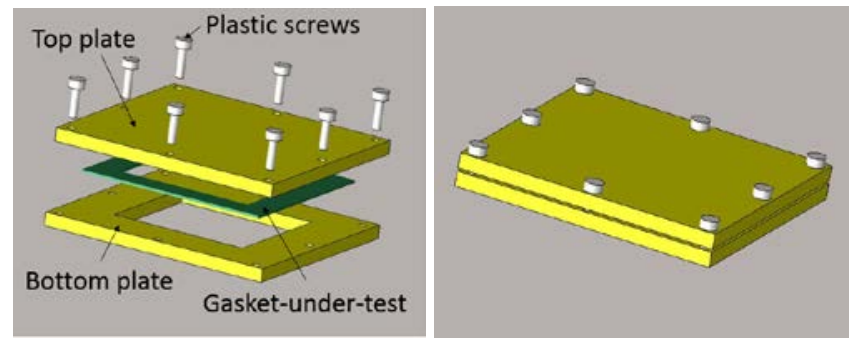

Fig. 5. Removable clamping module.

In order to overcome these difficulties, an adapted stripline set-up is proposed, as shown in Fig. 4. The main adaptation is the inclusion of a removable clamping module which continuously keeps the gasket-under-test under the same compression rate. Appropriate cut-outs in the bottom-plate (Fig. 6) and the clamping plate (Fig. 7) allow to easily mount the module in the set-up with high precision. Figure 5 shows the removable module in more detail. The gasket-under-test is compressed between a bottom plate, which has a square hole that fits over the PCB embedded in the ground plane of the stripline set-up, and a solid top plate. Small round plastic separators are used to control the compression rate, while plastic screws are used to compress the module and gasket.

However, due to the necessary hole in the bottom plate of the clamping module, parallel leakage could occur along the path underneath the clamping module. This would result in the fact that one is actually measuring this parallel leakage instead of the SE of the gasket-under-test. Therefore, it is crucial to close the gap underneath the clamping module with a highperformant gasket. In the remaining of the paper, we will refer to this gasket as the gasket-under-module. In practice, a silicon gasket is used and mounted inside an extra groove that is made in the cut-out of the bottom plate. Figure 6 shows a top-view of this cut-out, while Fig. 8 shows a cross-section along the middle of this cut-out. Finally, Fig. 9 shows a cross-section along the middle of the set-up when the clamping module is mounted in the set-up and compressed against the gasketunder-module. Note that no parallel leakage happens above the clamping module because of the solid top plate of this clamping module.

As will be shown in Section IV, the SE of the gasket-undermodule is a determining factor for the dynamic range of whole measurement the set-up. In essence, one cannot measure a SE that is better that the SE of this gasket-under-module.

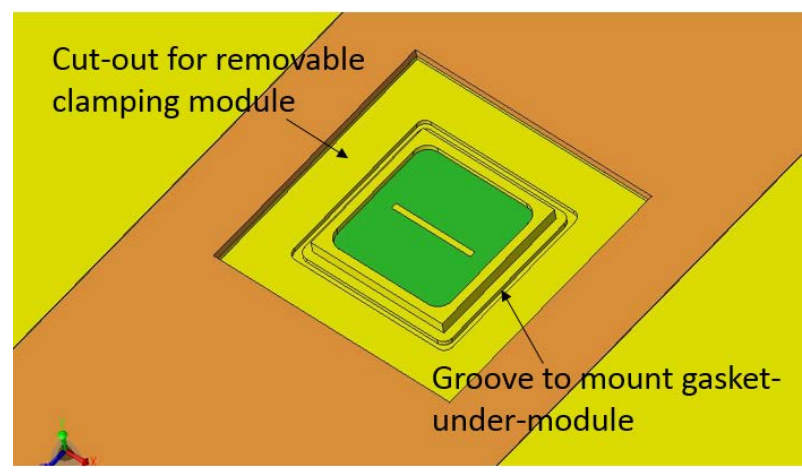

Fig. 6. Cut-out in bottom plate to fit removeable module, top-view.

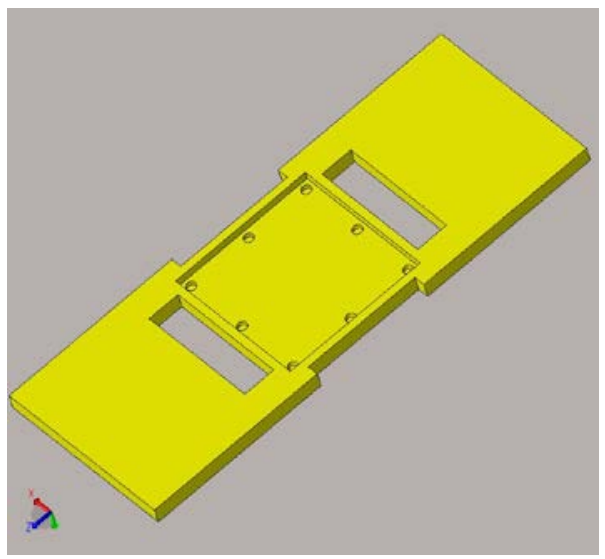

Fig. 7. Cut-out in top plate to fit removeable module.

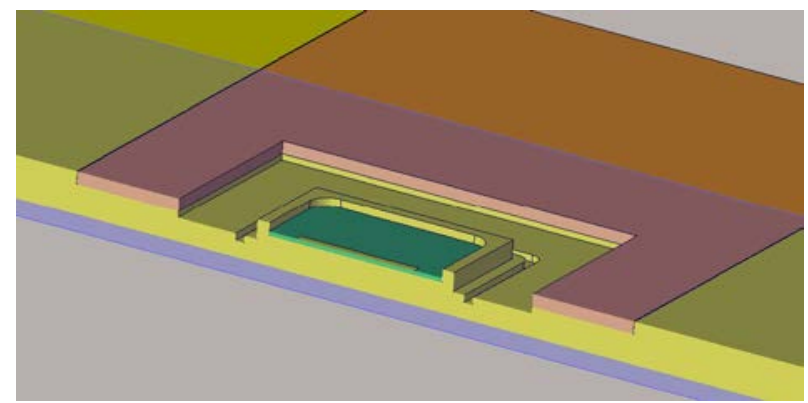

Fig. 8. Cut-out in bottom plate to fit removeable module, cross-section. 


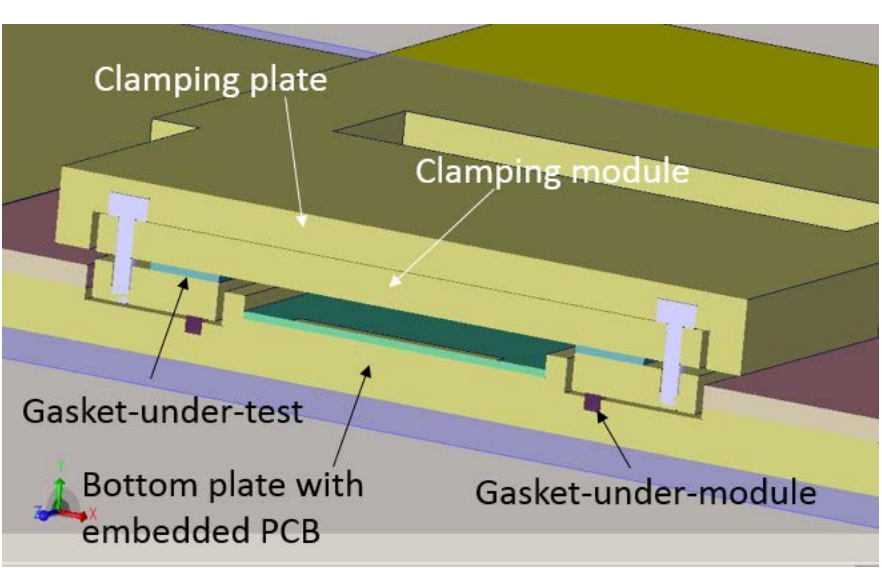

Fig. 9. Complete set-up, cross-section.

\section{DYNAMIC RANGE}

In this section, the influence of the gasket-under-module on the measured SE of the gasket-under-test is studied based on full-wave simulations of the adapted stripline set-up. In all examples below, the FDTD solver that is included in Keysight's 3D EM platform EMPro [10] was used. Both the gasket-under-test and the gasket-under-module are represented in a simplified way by assigning a given conductivity to their outer surface (the inner part of the gasket still being represented as air). As is shown in Fig. 9, a small gap was applied between the clamping module and clamping plate to also show that no parallel leakage occurs along that path above the clamping module.

The coupling between the left end of the microstrip and the left end of the stripline antenna is simulated and this for both the situation without and with the clamping module mounted in the set-up. From this the shielding effectiveness is calculated as indicated in eq. (1). A $3^{\text {rd }}$ order polynomial fit was applied to all SE curves, as [6] has shown that this agrees well with what would be measured in a reverberation room.

Four different situations are considered:

1. In the first situation, the gasket-under-test is modelled as a Perfectly Conducting (PEC) object, meaning that no leakage could occur along that gasket. The conductivity of the gasket-under-module is varied from $\sigma=0 \mathrm{~S} / \mathrm{m}$ (i.e. air or no gasket-under-module) to $\sigma=$ $10000 \mathrm{~S} / \mathrm{m}$. The resulting SE is shown in Fig. 10. Note that no gasket-under-module would result in an SE of OdB (i.e no shielding), while with a good gasket-undermodule it is possible to get to a high SE.

2. In the second situation, the conductivity of the gasketunder-test is varied from $\sigma=0 \mathrm{~S} / \mathrm{m}$ (i.e. air or no gasket-under-test) to $\sigma=10000 \mathrm{~S} / \mathrm{m}$. The gasket-undermodule is modelled as a PEC object meaning that no parallel leakage could occur underneath the clamping module. The resulting SE is shown in Fig. 11. From these results, we can conclude that if the gasket-undermodule is very performant (high SE), the set-up allows to accurately measure the SE of the gasket-under-test. Note that the levels of the SE agree very well with the $\mathrm{SE}$ values obtained in [6] for the normal stripline setup.

3. In the third situation, the conductivity of the gasketunder-test is varied from $\sigma=0 \mathrm{~S} / \mathrm{m}$ to $\sigma=10000 \mathrm{~S} / \mathrm{m}$. The gasket-under-module has a fixed conductivity of $\sigma$ $=100 \mathrm{~S} / \mathrm{m}$. The resulting SE is shown in Fig. 12. The same SE values are obtained as in Fig. 11 as long as the $\mathrm{SE}$ of the gasket-under-test is smaller than that of the gasket-under-module. All higher SE values are fixed to the one of the gasket-under-module.

4. In the fourth situation, the conductivity of the gasketunder-test is varied from $\sigma=0 \mathrm{~S} / \mathrm{m}$ to $\sigma=10000 \mathrm{~S} / \mathrm{m}$. The gasket-under-module has a fixed conductivity of $\sigma$ $=1000 \mathrm{~S} / \mathrm{m}$. The resulting SE is shown in Fig. 13. The maximal SE value of the gasket-under-test that can be measured is higher than in Fig. 12 thanks to the higher performance of the gasket-under-module.

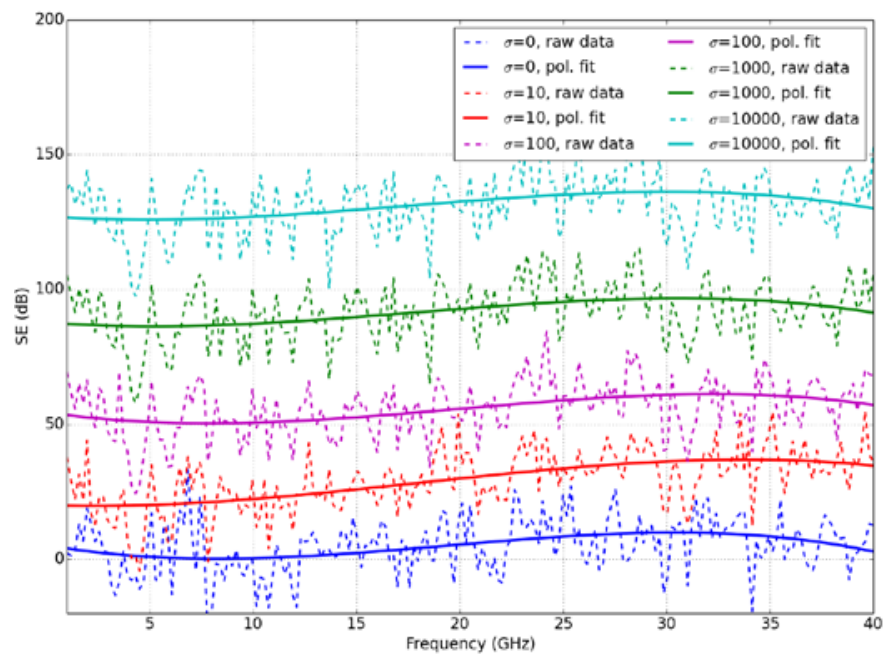

Fig. 10. Simulated SE for a PEC gasket-under-test and varying conductivity (unit $\mathrm{S} / \mathrm{m}$ ) for the gasket under the removeable clamping module.

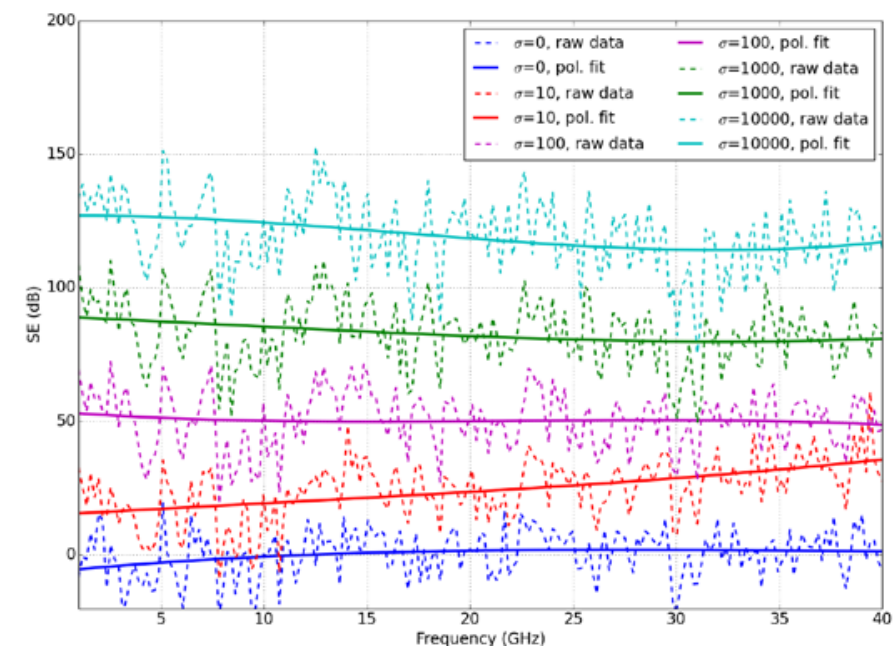

Fig. 11. Simulated SE for a gasket-under-test with varying conductivity (unit $\mathrm{S} / \mathrm{m}$ ) and a PEC gasket under the removeable clamping module. 


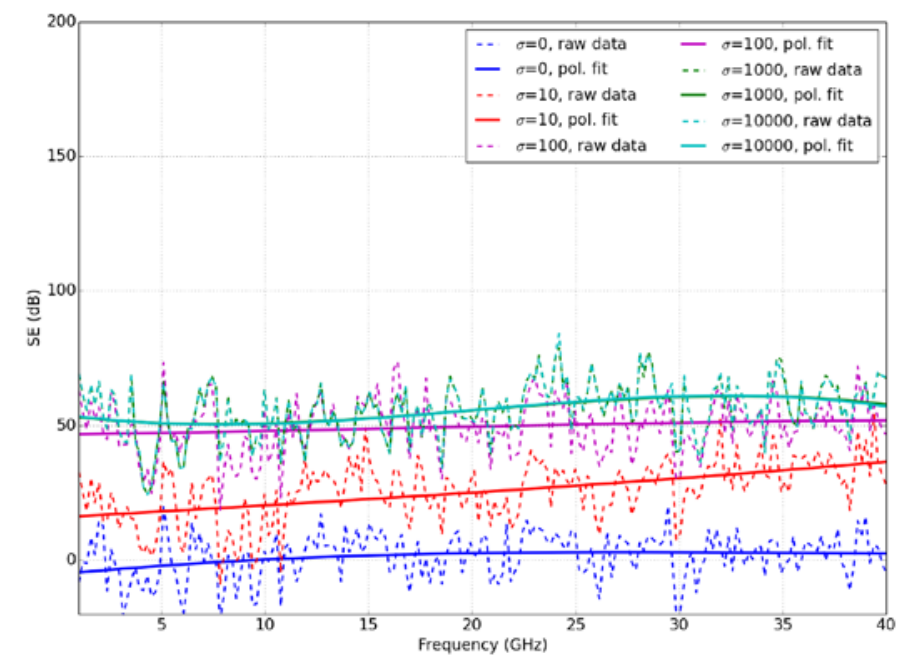

Fig. 12. Simulated SE for a gasket-under-test with varying conductivity (unit $\mathrm{S} / \mathrm{m}$ ) and a conductivity $=100 \mathrm{~S} / \mathrm{m}$ for the gasket under the removeable clamping module.

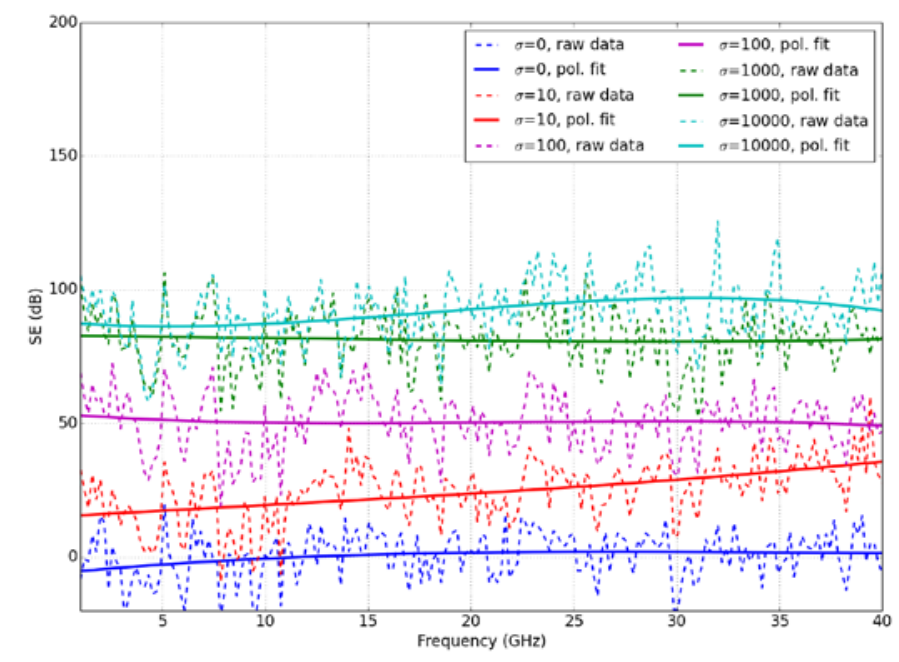

Fig. 13. Simulated SE for a gasket-under-test with varying conductivity (unit $\mathrm{S} / \mathrm{m}$ ) and a conductivity $=1000 \mathrm{~S} / \mathrm{m}$ for the gasket under the removeable clamping module.

It can be concluded that the performance of the gasketunder-module is the main determining factor for the dynamic range of the measurement set-up. In practice, a high performant silicon-based gasket is used.

\section{PRELIMINARY MEASUREMENT RESUltS}

A first set of measurements have been performed to validate the dynamic range of the new adapted stripline set-up and compare the obtained SE values with the ones measured with the normal stripline set-up. The measurements were done with a Vector Network Analyzer. A broadband 1 Watt amplifier (gain of $30 \mathrm{~dB}$ ) was used to maximize the power going into the stripline set-up. Port 1 of the VNA was feeding the structure (through the amplifier) at the left port of the embedded microstrip, while port 2 of the VNA was measuring the signal coming out of the set-up at the left port of the stripline antenna.

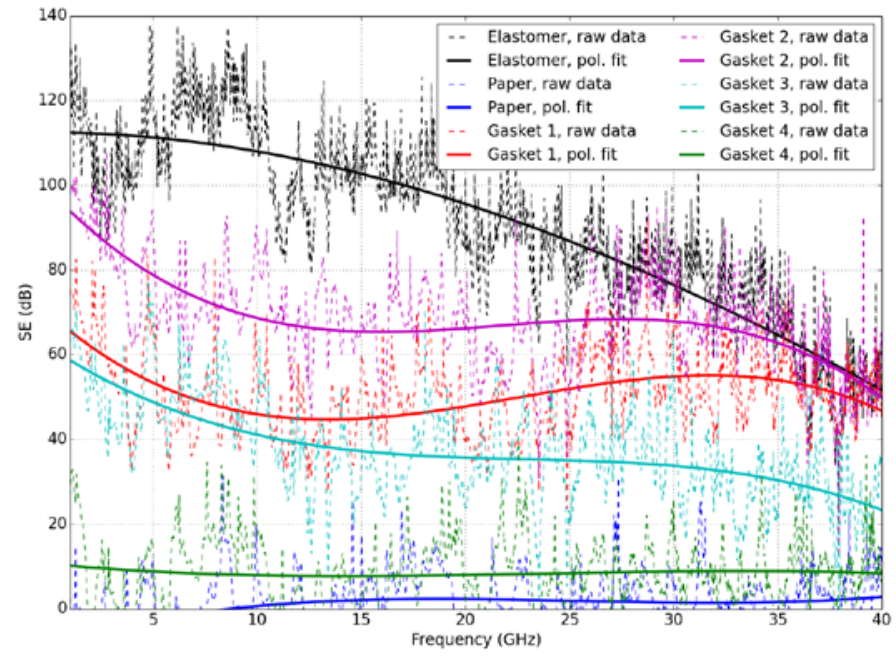

Fig. 14. Measured SE for different gaskets, adapted stripline set-up with removable clamping module

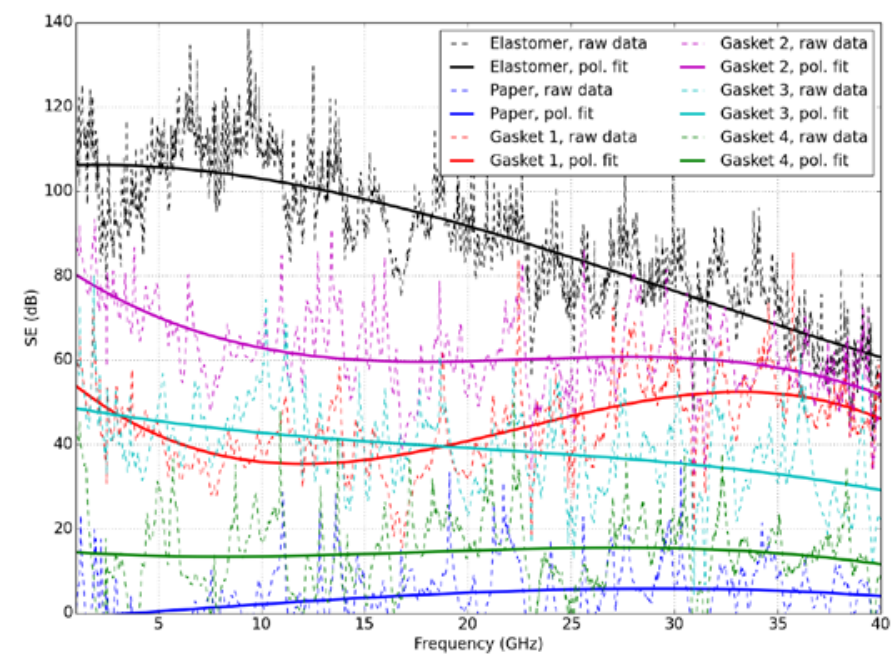

Fig. 15. Measured SE for different gaskets (the same as in Fig. 14), normal stripline set-up

Figures 14 and 15 give the measured SE values for the adapted and normal stripline set-up, respectively. The elastomer gasket is known to be very performant and gives an indication of the dynamic range that can be obtained. With both set-ups a dynamic range of around $100 \mathrm{~dB}$ at the lower frequencies and of around $60 \mathrm{~dB}$ at the higher frequencies is easily obtained. The similarity of the SE values measured with both stripline set-ups is very good. As an extra validation, the SE of a piece of paper was measured, resulting in a SE of OdB as it should be.

\section{CONCLUSION}

A novel measurement set-up dedicated at the characterization of the evolution of the high-frequency shielding-effectiveness of gaskets due to corrosion and ageing has been presented. The measurement set-up is based on a stripline set-up. Compared to the original stripline set-up, the adapted measurement set-up has a removable "clamping 
module" which can be easily mounted and removed from the set-up. The clamping module allows to age the gasket inside e.g. a climate chamber while always keeping the gasket under the same compression rate. Based on full-wave simulations, it was shown that the shielding-effectiveness given by this gasket underneath the clamping module is the main determining factor for the dynamic range of the set-up. A first set of measurements have proven the validity of the measurement setup and approach.

\section{ACKNOWLEDGMENT}

The authors would like to thank Yoeri Ariën of Laird Technologies for assistance with the measurements up to 40 $\mathrm{GHz}$.

\section{REFERENCES}

[1] SAE ARP 1705 rev. A - 2006, "Coaxial Test Procedure to Measure the RF Shielding Characteristics of EMI Gasket Materials"

[2] J. Catrysse, F. Vanhee, D. Pissoort, C. Brull, and G. Vandenbosch, "A new stripline measuring setup for the characterisation of conductive gaskets up to $18 \mathrm{GHz}$, Proceedings of the IEEE International Conference on Electromagnetic Compatibility, Fort Lauterdale, Florida, USA, July 2010, pp. 165-170

[3] J. Catrysse, F. Vanhee, D. Pissoort, C. Brull, and G. Vandenbosch, "Towards a global approach for the characterization of ICs and on board shielding components”, Proceedings of EMC Europe 2010, Wroclaw, Poland, September 2010, pp. 889-894

[4] J. Catrysse, F. Vanhee, D. Pissoort, C. Brull, and P. Reynaert, "Expanding the Stripline Measuring Set-up for the Characterisation of Conductive Gaskets up to $40 \mathrm{GHz}$ ", Proceedings of the IEEE International Conference on Electromagnetic Compatibility, Pittsburgh, Pennsylvania, USA, Aug. 2012, pp. 669-673

[5] J. Catrysse, F. Vanhee, D. Pissoort, C. Brull, and P. Reynaert, “A Measuring Setup for the Characterisation of 'in-circuit' Conductive Gaskets up to $40 \mathrm{GHz}$ ”, Proceedings of EMC Europe 2012, Rome, Italy, Sept. 2012

[6] D. Pissoort, B. Boesman., T. Claeys, J. Pitteman, J. Catrysse, "Correlating the High-Frequency Shielding Performance of 'On-Board' Gaskets when Characterized using a Stripline or Reverberation Room Method", Proceedings of the IEEE International Symposium on Electromagnetic Compatibility, Raleigh - North Carolina, USA, August 2014, pp. 128-133.

[7] B. Koerber et al., "IC-stripline: a new proposal for susceptibility and emission testing of IC's”, Proceedings EMC Compo 2007

[8] IEC 61967-8 ed.1, Integrated Circuits - measurements of electromagnetic emissions - part 8: measurement of radiated emissinons - IC stripline method

[9] IEC 62132-8 ed.1, Integrated Circuits - measurements of electromagnetic immunity - part 8: measurement of radiated immunity IC stripline method

[10] Keysight Technologies, EMPro, available from: http://www.keysight.com/ 